\title{
R\&D toward a Compact High-Brilliance X-ray Source based on Channeling Radiation
}

\author{
P. Piot ${ }^{* \dagger}$, C. A. Brau**, W. E. Gabella**, B. K. Choi ${ }^{\ddagger}$, , J. D. Jarvis ${ }^{* *}$, J. W. \\ Lewellen $^{\mathbb{Q}}$, M. H. Mendenhall** and D. Mihalcea* \\ *Northern Illinois Center for Accelerator \& Detector Development and Department of Physics, \\ Northern Illinois University, DeKalb, IL 60115, USA \\ ${ }^{\dagger}$ Accelerator Physics Center, Fermi National Accelerator Laboratory, Batavia, IL 60510, USA \\ ${ }^{* *}$ Department of Physics and Astronomy, Vanderbilt University, Nashville, TN 37235, USA \\ $¥$ Dept. of Electrical Engineering and Computer Science, Vanderbilt University, Nashville, TN 37235, USA \\ ${ }^{\S}$ Vanderbilt Institute of Nanoscale Science and Engineering, Vanderbilt University, Nashville, TN 37235, USA \\ II Physics Department \& Combat Systems, Naval Postgraduate School, Monterey, CA 93943, USA
}

\begin{abstract}
X-rays have been valuable to a large number of fields including Science, Medicine, and Security. Yet, the availability of a compact high-spectral brilliance X-ray sources is limited. A technique to produce X-rays with spectral brilliance $\mathscr{B} \sim 10^{12}$ photons. $(\mathrm{mm}-\mathrm{mrd})^{-2} \cdot(0.1 \% \mathrm{BW})^{-1} \cdot \mathrm{s}^{-1}$ is discussed. The method is based on the generation and acceleration of a low-emittance field-emitted electron bunches. The bunches are then focused on a diamond crystal thereby producing channeling radiation. In this paper, after presenting the overarching concept, we discuss the generation, acceleration and transport of the low-emittance bunches with parameters consistent with the production of high-brilliance X-rays through channeling radiation. We especially consider the example of the Advanced Superconducting Test Accelerator (ASTA) currently in construction at Fermilab where a proof-of-principle experiment is in preparation.
\end{abstract}

Keywords: beam dynamics, electron beams, X-rays

PACS: 29.27.-a, 41.85.-p, 41.75.F

\section{INTRODUCTION}

An electron propagating through a crystal can oscillate around the crystal's lattice plane. This process, referred to as planar channeling [1,2], can give rise to undulator-like radiation named channeling radiation (CR) $[3,4,5]$. The observation of CR was first reported in Ref. [6, 7, 8] and has since then been the object of intense researches $[9,10,11,12]$. The frequency of CR scales as $\omega=2 \gamma^{2} \omega_{0} /\left(1+\gamma^{2} \theta^{2}\right)$ where $\omega_{0} \propto \gamma^{-1 / 2}$ is the oscillation frequency around the crystal's lattice plane, $\gamma$ the Lorentz factor, and $\theta$ the observation angle with respect to the electron direction. Like inverse Compton scattering (ICS), CR can produce photons in the X-ray regime using moderate-energy electron beams (typically $20-50 \mathrm{MeV}$ ). Therefore, $\mathrm{CR}$ is a viable option for a compact X-ray source. In addition, CR is a relatively simple process to implement especially when compared with the complexity of the laser needed for ICS [13]. Although a precise description of CR properties relies a Quantum-mechanical description, the basic properties of $\mathrm{CR}$ can be inferred from a Classical treatment by considering the averaged potential barrier associated to the crystal. The properties of CR relevant to the concept discussed in this paper are briefly summarized below. In our study we plan to use a diamond crystal as channeling radiator because of its low $\mathrm{Z}$ and very high Debye temperature $(\sim 2000 \mathrm{~K})$.

A necessary condition for a particle to channel is that its transverse energy should be smaller that the effective potential barrier $U_{\text {eff }}$ associated to the crystal. This implies that the particle divergence should be smaller than the critical angle [2] $\psi_{c} \equiv \sqrt{U_{\text {eff }} / E}$, where $U_{\text {eff }}=1 / 2 \times U(0)$ with $U(0)$ being the average potential at zero distance from the plane. The critical angle depends on the crystal. For diamond, $\psi_{c} \sim 1 \mathrm{mrd}$ at $E \simeq 40 \mathrm{MeV}$. The condition for channeling radiation can be transposed to a requirement on the root-mean-square (rms) normalized beam emittance, e.g, $\varepsilon_{x} \equiv \frac{1}{m c}\left[\left\langle x^{2}\right\rangle\left\langle p_{x}^{2}\right\rangle-\left\langle x p_{x}\right\rangle^{2}\right]^{1 / 2}$ in the transverse phase space $\left(x, p_{x}\right)$ [here $\langle$.$\rangle represents the statistical averaging over$ the phase space distribution]. At the location of the crystal, i.e. where the beam is focused $\left(\left\langle x x^{\prime}\right\rangle=0\right)$, the requirement for the beam divergence to be smaller that $\psi_{c}$ implies that the rms beam spot on the crystal is $\sigma_{\perp} \equiv\left\langle x^{2}\right\rangle^{1 / 2}=\varepsilon_{x} /\left(\gamma \psi_{c}\right)$. The electron-beam transverse size $\sigma_{\perp}$ will also define the effective size of the X-ray source and therefore enters in the spectral brilliance since $\mathscr{B} \propto \sigma_{\perp}^{-2}$. Therefore, maximizing the brilliance calls for minimizing the beam emittance. In 
fact, targeting an average brilliance of $10^{12}$ photons. $(\mathrm{mm}-\mathrm{mrd})^{-2} \cdot(0.1 \% \mathrm{BW})^{-1} \cdot \mathrm{s}^{-1}$ requires a transverse emittance of $\varepsilon_{\perp} \simeq 1 \mathrm{~nm}$ [15]. Emittances within this order of magnitude have very recently been observed in photoinjectors [14]. Such emittances are also produced from field-emitter (FE) cathodes [16]. The latter type of cathodes presents the advantage of circumventing the use of a laser and are therefore a technology of choice for a compact and portable $\mathrm{X}$-ray source.

In this paper, after briefly outlining the proposed compact X-ray-source concept and its requirements, we explore the beam-dynamics aspects associated to the generation, acceleration and transport of nanometer-emittance electron beams. The beam dynamics studies are performed using the Advanced Superconducting Test Accelerator (ASTA) facility setup to demonstrate the possible use of this facility to support a proof-of-principle experiment aimed at producing $\sim 80-100-\mathrm{keV} \mathrm{X}$-rays via channeling radiation.

\section{OVERVIEW OF THE COMPACT X-RAY SOURCE}

A conceptual diagram of the proposed compact X-ray source appears in Fig. 1. Given the characteristic of CR, producing hard X-rays would require a beam energy of $\sim 20-40 \mathrm{MeV}$; see Fig. 1 (b). An RF gun with a FE cathode on its backplate produced the low-emittance electron bunches which are subsequently accelerated in a linear accelerator (linac). A set of quadrupole magnets focus the beam a diamond chip thereby producing the X-rays. The electron beam is separated from the X-rays and directed to a beam dump by a dipole magnet. The system could be made very compact. For instance using a X-band ( 2-cm long) 1-1/2 cell RF gun followed by a 50-cm long linac [17] would provide the needed beam parameters within a $\sim 1$-m length. More advanced acceleration concepts such as, e.g., microfabricated accelerators [18] could be used to further reduce the footprint of the system.
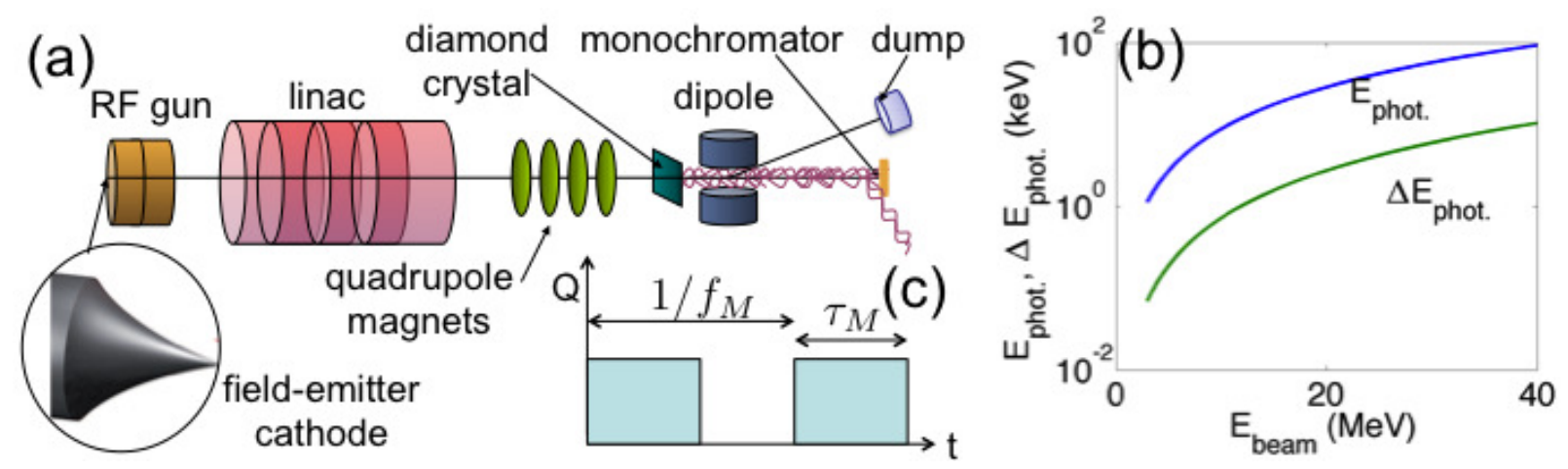

FIGURE 1. Overview of the compact X-ray source concept based on channeling radiation (a) showing the electron source (RF gun) linear accelerator (linac) and focusing optics (quadrupole magnets). For a pulsed RF system, the electron bunch are produced with frequency $f$ within macropulse of duration $\tau_{M}$ and frequency $f_{M}$ (b). The scaling of the X-ray energy $E_{p h o t}$ and energy dispersion $\Delta E_{\text {phot }}$ as function of electron beam energy $E_{\text {beam }}$ for a diamond cystal is shown in plot (c).

A parameter critical to imaging technique is the average spectral brilliance defined [in units of photons.(mm$\left.\mathrm{mrd})^{-2} \cdot(0.1 \% \mathrm{BW})^{-1} \cdot \mathrm{s}^{-1}\right]$ as

$$
\mathscr{B} \simeq \rho \frac{\gamma^{2} \psi_{c}^{2} N_{e} f \Upsilon_{e}}{\pi \varepsilon^{2}}
$$

where $N_{e}$ is the number of electron per bunch, $f$ the bunch repetition frequency, $\Upsilon_{e}$ the peak photon yield [in units of photons.(mm-mrd) $\left.)^{-2} \cdot(0.1 \% \mathrm{BW})^{-1} \cdot \mathrm{s}^{-1}\right]$, and $\rho \equiv f_{M} \tau_{m}$ is the duty cycle of the accelerator (where $f_{M}$ and $\tau_{M}$ are respectively the macropulse's frequency and duration; see also Fig. 1 [inset (c)]). The spectral average brilliance of the X-ray source is proportional to the average brightness of the electron beam which is a tradeoff between charge and frequency. A FE cathode typically operates at the linac frequency ( $\mathrm{GHz}$ regime) which considerably reduce the needed charge per bunch and therefore the associated beam emittance for a given brightness. 


\section{FIELD EMISSION CATHODES}

The FE cathode is a critical element for producing low-emittance beams. Over the last few years, Vanderbilt has had an active program and have demonstrated near-quantum-degenerate emission from individual field-emission tips at a per-tip current as high as $15 \mu \mathrm{A}[16,19]$. The FE cathodes are of two types, gated and ungated. Both types can be arranged as arrays. Ungated arrays, consisting simply of diamond pyramids, are more rugged and highly developed. Depending on the size and pitch of the pyramids, emission begins at macroscopic fields above about $5 \mathrm{MV} / \mathrm{m}$, and current density as high as $30 \mathrm{~A} . \mathrm{m}^{-2}$ has been obtained. Simulations and experiments show that the emittance of a 1 -mm cathode array is on the order of $1 \mu \mathrm{m}$. In gated FE cathodes, a self-aligned gate electrode is fabricated with the pyramids to control the emission. Emission typically turns on with a gate bias on the order of $50 \mathrm{~V}$, and only small macroscopic fields above the cathode are required to form a beam. The geometry of such an single-tip gated FE cathode is depicted in Fig. 2 (a) and (b).
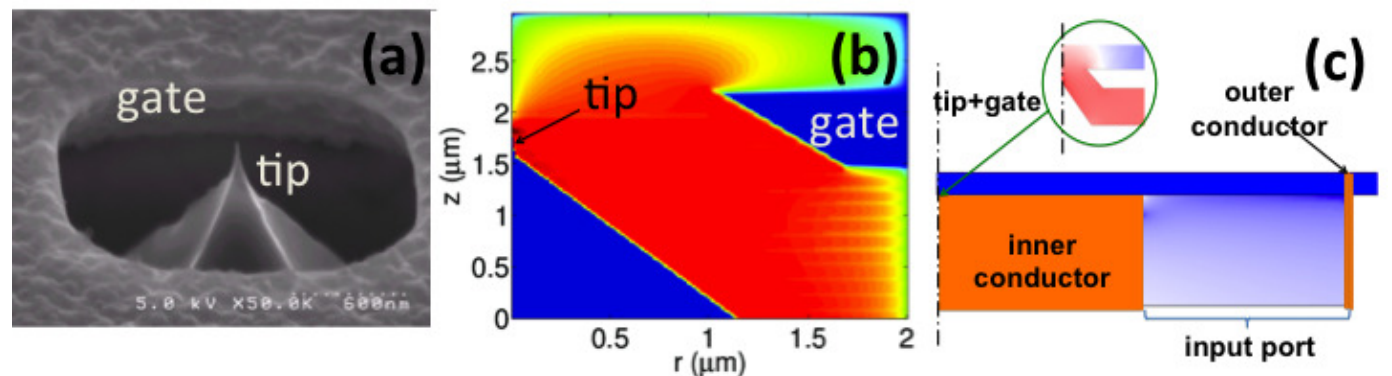

FIGURE 2. Electron-microscope photograph of the gated field emission cathode developed at Vanderbilt University (a) and corresponding simulated electrostatic field for a $70-\mathrm{V}$ potential difference between the gate and tip (the geometry is simplified to be cylindrically-symmetric and the field map is shown on a logarithm scale in the $(r, z)$ culindrical-coordinate system). Possible configuration of the gated FE cathode at the extremity of a coaxial line (the inner and outer conductors are shown in orange and the electromagnetic field simulations were performed with COMSOL [25]).

One of the main challenges is the formation of field-emitted bunches with durations much smaller than the RF accelerator wavelength. Such a duration is required to insure small energy spread is accumulated during acceleration. The bunch duration can be estimated by considering an FE cathode subject to a time-dependent electric field $E=E_{0} \cos (\omega t)$ with frequency $f \equiv \omega /(2 \pi)$. Such a configuration would field-emit an electron bunch with a temporal distribution that can be well approximated by Gaussian distribution with rms duration $\sigma_{t} \simeq \omega^{-1}\left[\beta E_{0} / B(\phi)\right]^{-1 / 2}$, Here we have considered the Fowler Nordheim's law $j=A(\phi) \beta^{2} E^{2} \exp [-B(\phi) /(\beta E)]$ with $A(\phi)$ and $B(\phi)$ being functions of the work function $\phi$ of the FE cathode material and $\beta$ is a field-enhancement factor; see Ref. [20, 21] for details. Therefore, one way of shortening the pulse is to use higher frequency, e.g., an harmonic frequency of the linac to be used to accelerated the field-emitted bunch. For an ungated FE cathode, a possible solution was developed in Ref. [22] where an RF gun supporting two harmonic modes was designed. In our approach we follow a similar technique but instead locate a gated FE cathode at the extremity of a coaxial line driven by two (and possibly more) harmonic RF fields; see configuration in Fig. 2 (c). Using a gated FE cathode in a RF gun provides an independent control of the emission phase that can be taken advantage to optimize the electron-beam dynamics.

The field emission process is simulated using the particle-in-cell (PIC) program IMPACT-T [23] to emit and track macroparticle in electrostatic field maps produced by the finite-difference program POISSON [24]. In order to gain confidence in these simulations we benchmarked our model of the FE cathode with simulations performed with the software CPO [26]. The typical transverse emittances simulated are 2.7 and $2.1 \mathrm{~nm}$ for respectively IMPACT-T and CPO models. Given the very different models, the observed agreement provides confidence that the generation of nanometer-level emittances is possible. Parametric studies performed with IMPACT-T indicate that space charge does not significantly affect the beam dynamics during the beam-generation stage for charge as high as $25 \mathrm{fC}$. For our required average current of $\langle I\rangle=200 \mathrm{nA}$, the needed charge per bunch is $q=\langle I\rangle / f \simeq 150 \mathrm{aC}$ for a $1.3-\mathrm{GHz} \mathrm{RF}$ system. 


\section{GENERATION AND TRANSPORT OF ULTRALOW-EMITTANCE BEAMS}

In this section we consider the acceleration and manipulation of the bunch produced by the single-tip gated FE cathode detailed in the previous section. We use the ASTA photoinjector configuration to carry our simulations; see Fig. 3. The electron source includes a 1.3-GHz RF gun with a coaxial RF input coupler [27]. Nominally, the gun incorporates a high-quantum-efficiency cesium telluride $\left(\mathrm{Cs}_{2} \mathrm{Te}\right)$ photocathode located on its back plate and illuminated by an ultraviolet laser pulse. The latter photocathode will eventually be replaced by the gated FE cathode to support the proposed X-rays channeling-radiation experiment. For a conservative peak electric field of $40 \mathrm{MV} / \mathrm{m}$ in the RF gun, the beam's mean energy is approximately $5 \mathrm{MeV}$ at the gun exit. The gun is surrounded by two identical solenoids (L1 and L2) which, under nominal operation, are used to correct for the space-charge-induced correlated emittance growth. Downstream of the RF gun, two TESLA-type superconducting cavities [28] (CAV1 and CAV2)

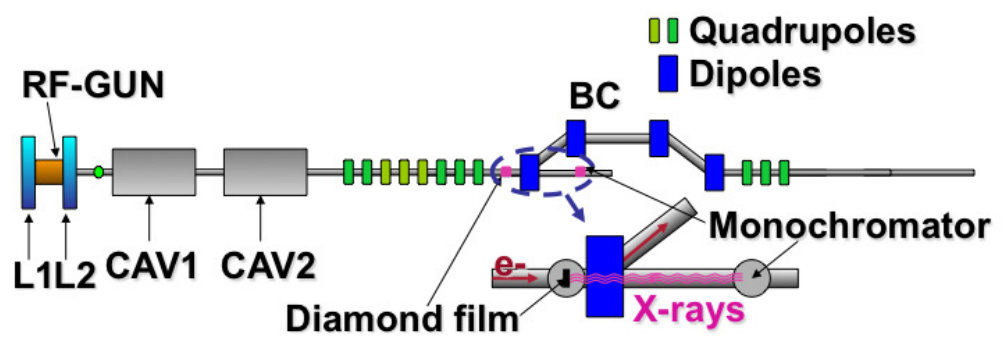

FIGURE 3. Overview of the low-energy injector. The legends are: "L1" "L2" solenoidal magnetic lenses, "CAV1" "CAV2" superconducting TESLA cavities, "BC" magnetic bunch compressor chicane. The green and blue rectangles respectively indicate the locations of quadrupole and dipole magnets.

accelerate the beam to $\sim 40 \mathrm{MeV}$. The downstream beamline includes generic focusing elements, instrumentations along with a round-to-flat beam transformer composed of three skew quadrupoles, and a magnetic bunch compressor (BC). The beam is then injected into a string of superconducting accelerating modules and accelerated to its final energy (eventually up to $\sim 800 \mathrm{MeV}$ ) [29]. The channeling-radiation experiment will be located upstream of the BC. In this configuration the electron beam will be bent by the first dipole magnet of the $\mathrm{BC}$ while the $\mathrm{X}$-rays will propagate to the detection system located straight-ahead; see Fig. 3.
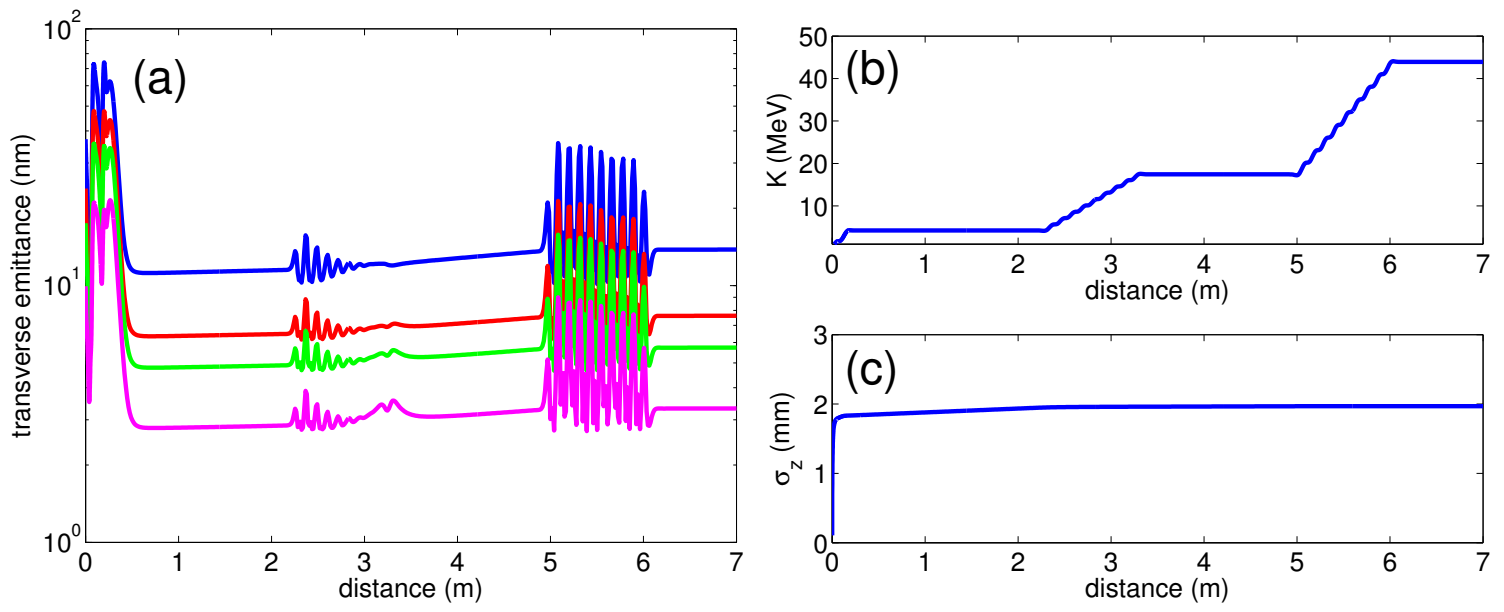

FIGURE 4. Transverse emittance (a), kinetic energy (b) and bunch length (c) evolution along the ASTA photoinjector accelerating section [comprising the RF gun $(0<z<30 \mathrm{~cm})$, CAV1 $(2.2<z<3.2 \mathrm{~m})$ and CAV2 $(5<z<6 \mathrm{~m})$ ]. The magenta, green, red and blue traces in plot (a) correspond to emittance respectively computed for the 85, 90, 95, and 100-percentile of the particle distribution.

The simulated macroparticle distribution downstream of the FE cathode is used as initial distribution for modeling the beam dynamics throughout the ASTA photoinjector. The simulation is segmented in two: the beam dynamics through the RF gun and acceleration is performed with the cylindrically-symmetric program ASTRA [30] that includes space-charge effects while the remaining of the beamline is simulated with the single-particle-dynamics program ELEGANT [31].The RF-gun, CAV1, and CAV2 are modeled by their axial electric fields obtained from SUPERFISH [24] 
simulations. All the cavities are assumed to be cylindrical-symmetric and the radial electric and azimuthal magnetic fields are derived form a third order expansion of Maxwell's equations in ASTRA. In Fig. 4 we present the evolution of the beam emittance from the FE cathode up to downstream of CAV2. The simulations were carried for a larger number of electron per bunch (correcponding to $25 \mathrm{fC}$ ), the total emittance is below 20 -nm while the $85 \%$-percentile emittance is approximately $3 \mathrm{~nm}$, a value that meets our requirements.

The upstream transport of the beam and focusing on the diamond crystal was optimized with ELEGANT including second-order effects. The transverse beam sizes along the accelerator beamline are displayed in Fig. 5 (a) and (b). At the location of the diamond crystal, the beam can be focused to a round spot with transverse full-width-half-maximum size of $\sim 5 \mu \mathrm{m}$; see Fig. 5 (b) and (c). Although this value is two orders of magnitude above the required beam size it was simulated with a much larger charge than required (our simulations were performed with $25 \mathrm{fC}$ while we only require $150 \mathrm{aC}$ ) and a statistical analysis of the transverse distribution confirms that the requirement of having 150 electron within a radius of $\sigma_{\perp}=40 \mathrm{~nm}$ is met. One remaining issue concerns the mitigation of the bremsstrahlung background produced by electron passing through the crystal but not channeling (due to their divergence being larger than $\psi_{c}$ ). Tracking simulations only based on first-order single-particle dynamics demonstrate that the generated
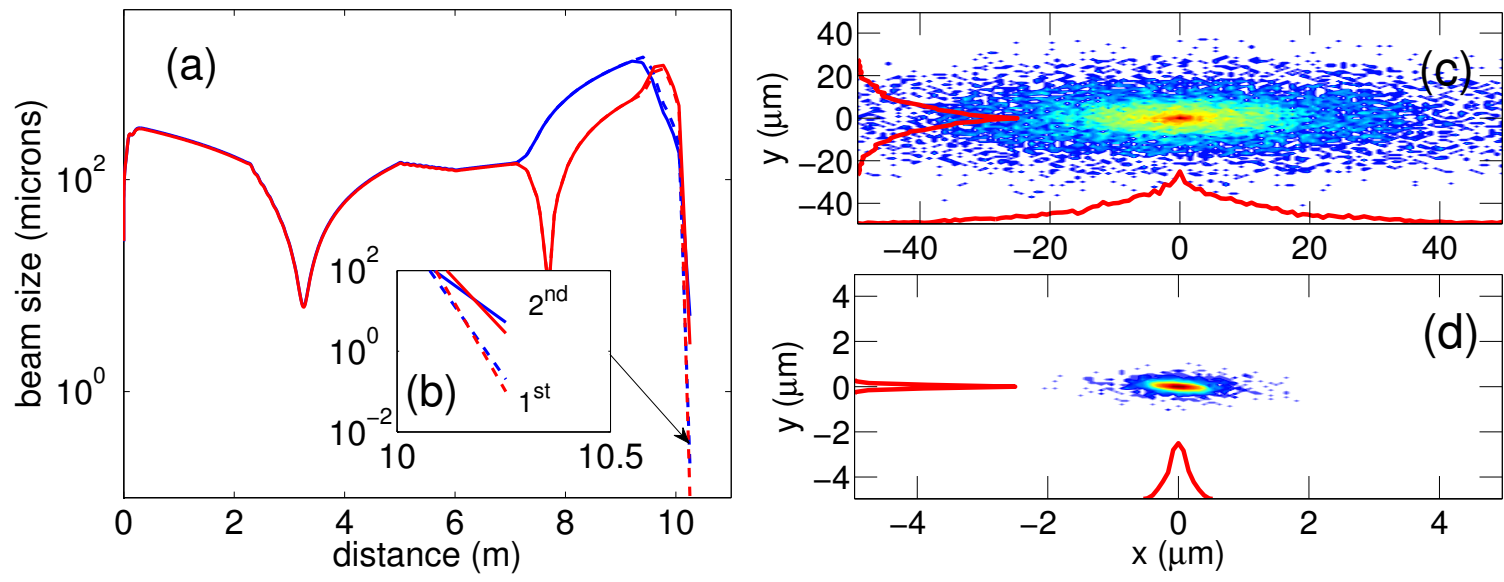

FIGURE 5. Horizontal (blue) and vertical (red) rms beam size evolution along the ASTA photoinjector beamline up to location of the diamond crystal (a). The solid and dashed line corresponds respectively to second- (" $2^{\text {nd }}$ ") and first-order (" $1{ }^{\text {st }}$ ") particletracking simulations and inset (b) shows the tms beam sizes in the vicinity of the crystal. Density plots (c) and (d) are respectively the beam transverse density at the crystal location obtained from second and first order calculations. The red trace in plots (c) and (d) are the corresponding horizontal and vertical projections.

emittance could results in beam size below $200 \mathrm{~nm}$ for a 25 -fC bunch charge; see Fig. 5 (d). The aberrations that lead to the larger spot size observed in Fig. 5 (c) are of chromatic nature and are a consequence of the rather long (2-mm rms) bunch produced by our gating technique. Such a long bunch acquires significant energy spread during acceleration in CAV1 and CAV2 as a result of the induced quadratic distortion of the longitudinal phase space by the cosine-like dependence of the RF accelerating voltage. The induced fractional energy spread, $\sigma_{\delta} \simeq 2 \times 10^{-3}$, plays a significant role on the transverse beam dynamics when trying to readch nanometer-scale spot sizes. A possible correction would include the addition of a harmonic RF cavity (operating at 3.9-GHz) to flatten the accelerating voltage across the bunch (thereby mitigating the quadratic distortion of longitudinal phase space). Such a solution is foreseen for the ASTA photoinjector design [32]. Another possible option would be the insertion of sextupole magnets to reduce the chromaticity of the lattice used to focus the beam. In the short term, a simpler method consists in energy-collimating the beam upstream of the diamond crystal.

\section{SUMMARY}

In this paper we have considered channeling radiation driven by low-emittance beams produced by field-emitter cathodes as a possible path toward the realization of a compact, portable, high-spectral-brilliance X-ray source. Startto-end simulations of the beam dynamics indicate that producing, accelerating such a beam while preserving the low 
emittance is possible. A channeling-radiation experiment is currently in preparation at the ASTA facility at Fermilab and its details will be reported elsewhere [33].

\section{ACKNOWLEDGMENTS}

This work was supported by the DARPA Axis program under contract AXIS N66001-11-1-4196 with Vanderbilt University and Northern Illinois University. The work of P.P. is partially supported by the Fermi Research Alliance, LLC under Contract No. DE-AC02-07CH11359 with the U.S. Department of Energy. We are thankful to R. A. Carrigan, Jr. for useful discussions on channeling radiation and to M. Church and V. Shiltsev for their support.

\section{REFERENCES}

1. J. Lindhard, Phys. Lett. 12126 (1964).

2. J. Lindhard, Mat. Fys. Medd. Dan. Vidensk. Selsk. 3414 (1965).

3. M. A. Kumakhov, Phys. Lett. A 57, 17 (1976).

4. M. A. Kumakhov, Sov. Phys. JETP 45 (4), 781 (1978).

5. M. A. Kumakhov, Radiation Effects and Defects in Solids null, 329 (1994).

6. $\quad$ R. W. Terhune and R. H. Pantell, Appl. Phys. Lett. 30, 265 (1977).

7. C. K. Gary, R. H. Pantell, M. Özcan, M. A. Piestrup, and D. G. Boyers, J. Appl. Phys. 70, 2995 (1991).

8. R. K. Klein, et al, Phys. Rev. B 31, 68 (1985).

9. J. U. Andersen, E. Bonderup, R. H. Pantell, Ann. Rev. Nucl. Sci 33453 (1983).

10. L.A. Gevorgian, K.A. Ispirian, R.K. Ispirian, Nucl. Instr. Meth. B 145155 (1998).

11. R. A. Carrigan, Jr., J. Freudenberger, S. Fritzler, H. Genz, A. Richter, A. Ushakov, A. Zilges, and J. P. F. Sellschop, Phys. Rev A 68, 062901 (2003).

12. W. Wagner, B. Azadegan, M. Sobiella, J. Steiner, K. Zeil, J. Pawelke, Nucl. Instr. Meth. B 266327 (2007).

13. R. A. Carrigan, Jr., M.Kh. Khokonov, "Channeling radiation as virtual Thomson scattering and the relative efficiency of X-ray production by intense laser beams", preprint (unpublished) FERMILAB-PUB-97-115 (1997).

14. R. Li, et al., these proceedings [contribution to Working Group 5 of the 2012 Advanced Accelerator Concept workshop (AAC12)].

15. C. A. Brau, B.-K. Choi, J. D. Jarvis, J. W. Lewellen, and P. Piot, Synchrotron Radiation News 25 (1), 20 (2012).

16. J. D. Jarvis, H. L. Andrews, B. Ivanov, C. L. Stewar, N. de Jonge, E. C. Heeres, W.-P. Kang, Y.-M. Wong, J. L. Davidson, and C. A. Brau, J. Appl. Phys. 108, 094322 (2010).

17. F. Wang, C. Adolphsen, and C. Nantista Phys. Rev. ST Accel. Beams 14, 010401 (2011).

18. A. Nassiri, at al., Proc. Int. Electron Devices Meeting, Washington, DC, December 1993.

19. J. D. Jarvis, H. L. Andrews, C. A. Brau, B. K. Choi, J. Davidson, W. P. Kang, Y. M. Wong, J. Vac. Sci. Technol. B 27, 2264 (2009).

20. J. W. Wang and G. A. Loew, "Field Emission and RF breakdown in high-gradient room-temperature linac structure", report SLAC-PUB-7684 (1997).

21. D.Y. Zhong, G.Y. Zhang, S. Liu, T. Sakurai, E.G. Wang, Appl. Phys. Lett. 80506 (2002).

22. J. W. Lewellen and J. Noonan, Phys. Rev. ST Accel. Beams, 8, 033502 (2005).

23. J. Qiang, S. Lidia, R. D. Ryne and C. Limborg-Deprey, Phys. Rev. ST Accel. Beams 9, 044204 (2006).

24. J. H. Billen and L. M. Young, Proceedings of the 1993 Particle Accelerator Conference (PAC93), Washington DC (IEEE, Piscataway, NJ, 1993), 790 (1993).

25. COMSOL Multiphysics Finite Element Analysis Software version 4.3 (2012).

26. The CPO charged particle optics program is commercially available from CPO Ltd. Charged Particle Optics programs.

27. B. Dwersteg, K. Flöttmann, J. Sekutowicz, Ch. Stolzenburg, Nucl. Instr. and Meth. A 393, 93 (1997).

28. B. Aune et al., Phys. Rev. ST Accel. Beams 3, 092001 (2000).

29. M. Church, S.Nagaitsev, and P. Piot, Proc. Proceedings of the 2007 Particle Accelerator Conference (PAC07), Albuquerque NM, 2942 (2007).

30. K. Flöttmann, http://www.desy.de/ mpyflo.

31. M. Borland, Advanced Photon Source report LS-287, Argonne IL, September 2000 (unpublished).

32. P. Piot, Y.-E Sun and M. Church, Proceedings of the 2010 International Particle Accelerator Conference (IPAC10), Kyoto, Japan, 436 (2010).

33. W. E. Gabella, C. A. Brau, B. Choi, B. Ivanov, J. D. Jarvis, J. Lewellen, M. H. Mendenhall, D. Mihalcea, M. Moss, and P. Piot, "Generation and Application of Channeling X-Rays using a Novel Low-Emittance Electron Beam: Plans and Status", to be presented at the 5th International Conference on Charged and Neutral Particles Channeling Phenomena (channeling 2012), September 23-28, 2012, Alghero (SS), Italy. 